\title{
Comparative Evaluation of Linear- Rotary Actuator Topologies for Highly Dynamic Applications
}

\section{Conference Paper}

Author(s):

Mirić, Spasoje; Tüysüz, Arda; Kolar, Johann W. (D)

Publication date:

2017

Permanent link:

https://doi.org/10.3929/ethz-b-000187556

Rights / license:

In Copyright - Non-Commercial Use Permitted

Originally published in:

https://doi.org/10.1109/IEMDC.2017.8002236 


\title{
Comparative Evaluation of Linear-Rotary Actuator Topologies for Highly Dynamic Applications
}

\author{
Spasoje Mirić, Arda Tüysüz and Johann W. Kolar \\ Power Electronic Systems Laboratory \\ ETH Zürich \\ Zürich, Switzerland \\ Email: miric@lem.ee.ethz.ch
}

\begin{abstract}
A Linear-Rotary Actuator (LiRA) is an electrical machine whose mover can move linearly and rotate as a direct drive (without mechanical transmissions/gearboxes). Such actuators are successfully used in various applications such as pickand-place machines that mount components on Printed Circuit Boards (PCBs), servo actuation of gearboxes, and in robot arms and/or end effectors. In order to design a compact LiRA with high dynamics, two different LiRA topologies are compared in this paper. A fair comparison is obtained by fixing the outer dimensions of the examined LiRA topologies (diameter and length). Electrical loading of the machines is determined using a simple thermal model, and an optimization based on finite element analysis is carried out. Performance criteria, such as circumferential and axial accelerations as well as maximum axial forces of all analyzed machines are compared. Moreover, metrics like total machine mass and volume of the used permanent magnets are included in the evaluation.
\end{abstract}

Keywords- Finite-Element Method, Linear-Rotary Actuator, Magnetic Design, Multi-Objective Optimization, 2DoF Actuator.

\section{INTRODUCTION}

Electromagnetic actuators that can move linearly and rotate are widely used in different applications such as pick-andplace machines that mount components on Printed Circuit Boards (PCBs) [1], servo actuation of gearboxes [2], or in robotics as arms or end effectors [3]. Conceptually, a LinearRotary Actuator (LiRA) can be realized by mechanical or magnetic coupling of linear and rotary electrical machines [1], [4]. Mechanical coupling can be realized with an arrangement where one machine, e.g. the linear machine, moves the whole rotary machine (rotor and stator), or vice versa [1]. This, however, limits the overall dynamics and efficiency of the system, due to the increased moving mass. Alternatively, linear and rotary machines may be connected on the same shaft in an attempt to reduce the moving mass [1]; nevertheless, the mover of each machine needs to be at least twice the axial stroke longer than the stator in both linear and rotary machines in order to maintain a constant active length.

Another conceptual approach to build a LiRA is by using two co-axial stators that are displaced radially instead of axially (e.g. one inside another) [5], where e.g. the inner

This work was supported by ETH Research Grant ETH-13 16-2. part is the rotary machine and the outer part is the linear machine. In this arrangement, the linear and rotary machines are magnetically decoupled by the back iron (magnetic yoke) of the mover. Conversely, the actuator topology analyzed in [4] is an example of a complete magnetic coupling of a linear and rotary machine, i.e. the same magnetic materials are used for both movements. In both cases, the mover has to be only one axial stroke longer than the stator [4], [5].

Similar to any other electromechanical actuator, a LiRA can be realized using a slotless or a slotted stator. The slotless topology features several advantages such as practically no load-dependent magnetic saturation of the core, no rotor losses caused by slot harmonics [6], and no cogging torque/force. Slotted machine topologies, on the other hand, are usually characterized by higher torque/force densities. Hence, LiRAs with slotted stators are likely to feature higher acceleration capabilities. Therefore, actuator analysis starts with slotless designs and continues with the introduction of slots, aiming for higher torque/force.

In this paper, two LiRA topologies that can yield compact design are compared: a magnetically coupled machine, cf. Fig. 1, and a machine with two stators, cf. Fig. 5, henceforth called Topology A and B, respectively. For comparing the machines the achievable circumferential (rotational) and axial (linear) accelerations, total actuator mass, total permanent magnet (PM) volume, and the continuous axial force are chosen as performance indicators.

Following this brief introduction, Sec. II introduces geometry parametrization and optimization approach of the topologies. Modeling approach and simulation results for Topology A and Topology B are included in Sec. III and Sec. IV, respectively. The comparison of the two topologies is conducted in Sec. V. Sec. VI concludes the paper.

\section{GeOMETRY PARAMETRIZATION AND Optimization}

In order to obtain a fair comparison of LiRA topologies A and $\mathrm{B}$, the outer dimensions (outer diameter and axial length of the stator) of the actuators are fixed to the same values. Within this limited volume, geometries of the actuators are optimized for maximizing the circumferential and axial accelerations as well as the continuous axial force. At the beginning of the optimization process, the geometries are parametrized 
TABLE I: Topology A (cf. Fig. 1) discrete geometric parameter set where: $\varphi_{\tau}=2 \pi / N_{\mathrm{pm}, \varphi}, z_{\tau}=L / N_{\mathrm{pm}, z}$ and $z_{\text {coil }}=L / N_{\text {coil }, z}$.

\begin{tabular}{|c|c|c|}
\hline Symbol & Parameter name & Values \\
\hline \multicolumn{3}{|c|}{ Swept parameters } \\
\hline$R_{\text {in }}$ & mover inner radius & $4,8,12(\mathrm{~mm})$ \\
\hline$r_{\mathrm{pm}}$ & PM thickness & $2,5,8(\mathrm{~mm})$ \\
\hline$\varphi_{\mathrm{pm}}$ & $\mathrm{PM} \varphi$ size & $0.5,0.75,1 \times \varphi_{\tau}$ \\
\hline$z_{\mathrm{pm}}$ & $\mathrm{PM} z$ size & $0.5,0.75,1 \times z_{\tau}$ \\
\hline$z_{\mathrm{Cu}}$ & conductor axial thickness & $0.2,0.4,0.6,0.8 \times z_{\text {coil }} / 2$ \\
\hline$N_{\mathrm{pm}, \varphi}$ & pole number, tangential & 4,8 \\
\hline$N_{\mathrm{pm}, \mathrm{z}}$ & pole number, axial & 4,8 \\
\hline$N_{\text {coil,z }}$ & coil number, axial & 6,9 \\
\hline \multicolumn{3}{|c|}{ Fixed parameters } \\
\hline$R_{\text {out }}$ & outer radius & $25(\mathrm{~mm})$ \\
\hline$L$ & actuator length & $120(\mathrm{~mm})$ \\
\hline$r_{\mathrm{ag}}$ & air gap & $0.5(\mathrm{~mm})$ \\
\hline$r_{\mathrm{f}}$ & coil former thickness & $0.5(\mathrm{~mm})$ \\
\hline$r_{\mathrm{m}}$ & mover iron thickness & $2(\mathrm{~mm})$ \\
\hline$r_{\mathrm{s}}$ & stator iron thickness & $2(\mathrm{~mm})$ \\
\hline$N_{\text {coil }, \varphi}$ & coil number, tangential & 6 \\
\hline
\end{tabular}

according to Fig. 1 and Fig. 5. Then, the geometric parameters are discretized in accordance with Tab. I and Tab. III.

For each geometry combination, two magnetostatic Finite Element Method (FEM) simulations are run where: (1) torque and circumferential acceleration and (2) axial force and acceleration are calculated for given copper losses. The electrical loading in these simulations is based on a thermal model, which is detailed in the following section.

The selected method of analysis finds the global optimum (or a Pareto-optimum set of designs) over the discrete design space, and there is no need for defining a cost function to evaluate the impact of usually competing design goals such as force-per-copper-loss and mass, which would be required when conventional optimization methods would be used [7].

\section{TOPOLOGY A}

This topology is shown in Fig. 1. Its mover, which can move linearly and rotate, is equipped with radially magnetized PMs whose magnetization directions (radially inwards or radially outwards) are altered both in circumferential and axial directions. A set of concentrated coils in the stator allows winding currents to control the torque and axial force independently.

This topology is analyzed both with slotless and slotted stator variants. The analysis starts with a slotless stator, as in this case there is no saturation in the actuator, and no losses are caused by slot harmonics [6], and no cogging is present.

The lumped-parameter thermal equivalent circuit shown in Fig. 2 is used to calculate maximum copper losses, such that the maximum temperature of the windings does not exceed $T_{\mathrm{w}, \max }=120^{\circ} \mathrm{C}$. The ambient temperature is $T_{\mathrm{amb}}=25^{\circ} \mathrm{C}$, and forced air cooling with an air speed of $2.7 \mathrm{~m} \mathrm{~s}^{-1}$ is assumed around the cylindrical stator. Only copper losses are considered at the first step, which is well justified for the slotless stator type. The resistances in the circuit refer to the radial-direction thermal resistances of a single coil $R_{\mathrm{Cu}}^{\mathrm{T}}$, coil former $R_{\mathrm{f}}^{\mathrm{T}}$, stator back iron $R_{\mathrm{Fe}}^{\mathrm{T}}$ and stator-to-air thermal resitance $R_{\mathrm{s}-\mathrm{a}}^{\mathrm{T}}$. Values of the thermal resistances for an example geometry with $N_{\text {coil }}=36$ calculated according

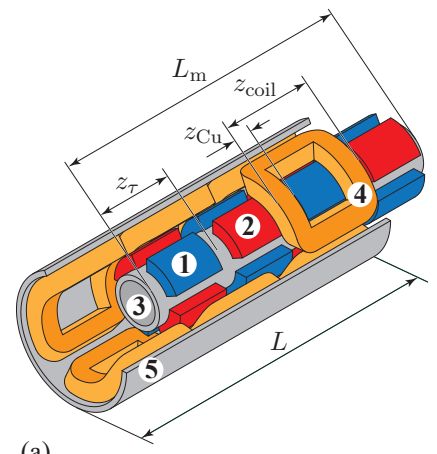

(a)

Fig. 1: LiRA Topology A: (a) 3-D cross-section with geometry parameters in $z$ direction; mover: (1) south PM pole, (2) north PM pole, (3) mover back iron; stator: (4) coils, (5) stator back iron (b) radial cross-section with geometry parameters in $r, \varphi$ directions.

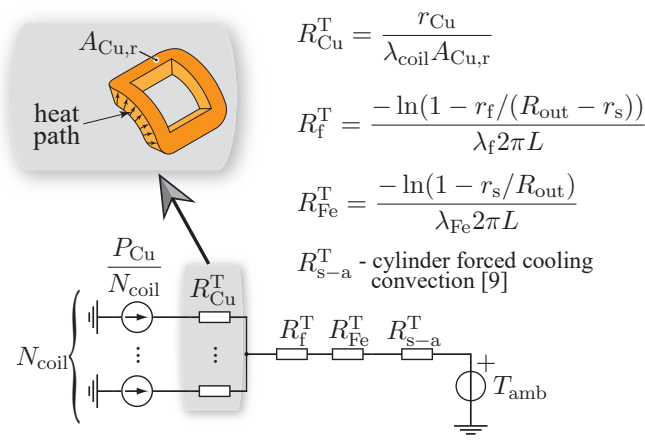

Fig. 2: LiRA Topology A lumped-parameter thermal model, where: $\quad \lambda_{\text {coil }}=2 \mathrm{~W} \mathrm{~m}^{-1} \mathrm{~K}^{-1}, \quad \lambda_{\mathrm{f}}=0.1 \mathrm{~W} \mathrm{~m}^{-1} \mathrm{~K}^{-1}$ and $\lambda_{\mathrm{Fe}}=22 \mathrm{~W} \mathrm{~m}^{-1} \mathrm{~K}^{-1}$ (laminated steel, [8]).

to Fig. 2 are: $R_{\mathrm{Cu}}^{\mathrm{T}}=10.89 \mathrm{~K} \mathrm{~W}^{-1}$ per coil (36 coils are in parallel), $R_{\mathrm{f}}^{\mathrm{T}}=0.78 \mathrm{~K} \mathrm{~W}^{-1}$ and $R_{\mathrm{Fe}}^{\mathrm{T}}=0.01 \mathrm{~K} \mathrm{~W}^{-1}$. Using Hilpert's correlation [9], the average heat transfer coefficient from the stator to the air is calculated to be $23.9 \mathrm{~W} \mathrm{~m}^{-2} \mathrm{~K}^{-1}$, which yields $R_{\mathrm{s}-\mathrm{a}}^{\mathrm{T}}=2.67 \mathrm{KW}^{-1}$. Using these values, the winding temperature of $\approx 120^{\circ} \mathrm{C}$ is reached with $P_{\mathrm{Cu}}=25 \mathrm{~W}$ in the windings.

The analysis of the established thermal network reveals that the highest contributor to the winding temperature is the statorto-air thermal resistance $R_{\mathrm{s}-\mathrm{a}}^{\mathrm{T}}$. This justifies the assumption that allowed losses in the actuator depend only on the $R_{\mathrm{s}-\mathrm{a}}^{\mathrm{T}}$. As $R_{\mathrm{s}-\mathrm{a}}^{\mathrm{T}}$ depends only on the outer dimensions of the actuator, which are fixed in the optimization process, copper losses can also be fixed in the optimization.

In order to evaluate the performance of the slotless designs of Topology A, an electromagnetic model is established, where first the PM field $\vec{B}_{\mathrm{PM}}$ in the winding region is obtained using 3-D FEM analysis, and then the torque and axial force are calculated as

$$
\vec{T}=\int_{V_{\text {coil }}} \vec{r} \times\left(\vec{J}_{\max } \times \vec{B}_{\mathrm{PM}}\right) \mathrm{d} r r \mathrm{~d} \varphi \mathrm{d} z,
$$


TABLE II: Parameters of the slotless Topology A candidate designs with radially magnetized PMs (A.I) and with Halbach PM arrangement (A.II) in the mover.

\begin{tabular}{l|ccccl}
\hline \hline & \multicolumn{5}{|c}{ Radial A.I/Halbach A.II } \\
Par./Unit & $\mathbf{( 1 )}$ & $\mathbf{( 2 )}$ & $\mathbf{( 3 )}$ & $\mathbf{( 4 )}$ & \\
\hline$R_{\text {in }}(\mathrm{mm})$ & $4 / 4$ & $4 / 4$ & $4 / 8$ & $8 / 12$ & \\
$r_{\mathrm{pm}}(\mathrm{mm})$ & $2 / 2$ & $2 / 2$ & $5 / 2$ & $5 / 2$ & \\
$\varphi_{\mathrm{pm}}$ & $0.75 / 0.25$ & $0.75 / 0.25$ & $0.5 / 0.5$ & $0.75 / 0.5$ & $\times \varphi_{\tau}$ \\
$z_{\mathrm{pm}}$ & $0.75 / 0.5$ & $0.75 / 0.5$ & $0.75 / 0.5$ & $0.75 / 0.5$ & $\times z_{\tau}$ \\
$z_{\mathrm{Cu}}$ & $0.6 / 0.4$ & $0.6 / 0.6$ & $0.6 / 0.6$ & $0.6 / 0.6$ & $\times \frac{z_{\text {coil }}}{2}$ \\
$N_{\mathrm{pm}, \varphi}$ & $4 / 4$ & $4 / 4$ & $4 / 4$ & $4 / 4$ & \\
$N_{\mathrm{pm}, \mathrm{z}}$ & $4 / 4$ & $8 / 8$ & $8 / 8$ & $8 / 8$ & \\
$N_{\text {coil }, \mathrm{z}}$ & $6 / 6$ & $6 / 6$ & $6 / 6$ & $6 / 6$ & \\
\hline
\end{tabular}

and

$$
\vec{F}=\int_{V_{\text {coil }}}\left(\vec{J}_{\text {max }} \times \vec{B}_{\mathrm{PM}}\right) \mathrm{d} r r \mathrm{~d} \varphi \mathrm{d} z
$$

for the same $\vec{B}_{\mathrm{PM}}$. Hence, taking advantage of the slotless design, the torques and axial forces of the machines sharing the same rotor (and stator core) design can be evaluated as postprocessing steps (each with electric loading leading to $25 \mathrm{~W}$ copper losses) after a single solution of the 3-D FEM model. In the final step, physical properties (volume, mass, moment of inertia) and achievable accelerations (circumferential $a_{\varphi}$ and axial $a_{\mathrm{z}}$ ) are calculated.

Two different movers are considered for the LiRA Topology A: (1) Topology A.I with radially magnetized PMs in the mover, shown in Fig. 1(a), and (2) Topology A.II with a Halbach PM array in the mover [10]. In Topology A.II, there is no back iron in the mover, which allows for a lighter mover and consequently, higher accelerations. The PM thickness is equal to $r_{\mathrm{pm}}^{\prime}=r_{\mathrm{m}}+r_{\mathrm{pm}}$, cf. Tab. I.

The achievable circumferential and axial accelerations as well as the axial force for slotless actuator Topologies A.I and A.II are plotted in Fig. 3(a) and Fig. 3(b), respectively. A contradiction between the axial force and the achievable accelerations can be observed in the results, as a higher volume of the PMs in the mover produces a stronger field and yields higher force, but at the same time increases the mover's mass and moment of inertia, which is detrimental for accelerations. Therefore, a trade-off can be found by varying the PMs width and thickness $\left(\varphi_{\mathrm{pm}}, z_{\mathrm{pm}}\right.$ and $r_{\mathrm{pm}}$, cf. Tab. I).

The optimum designs are the ones on the Pareto front, which is defined as the selection of designs, where one performance metric (e.g. axial acceleration) cannot be increased further without sacrificing another performance metric (e.g. circumferential acceleration). Four candidate designs of slotless Topologies A.I and A.II that yield different optima are chosen and their geometrical parameters are given in Tab. II.

A further improvement of the chosen designs is achieved with adding teeth (slots) to the slotless Topology A candidate designs. Two types of slots are considered: open slots and semi-closed slots that are shown in Fig. 4. Since there is a risk of teeth saturation, each of these designs is checked with 3-D FEM. The introduction of the slots significantly improves

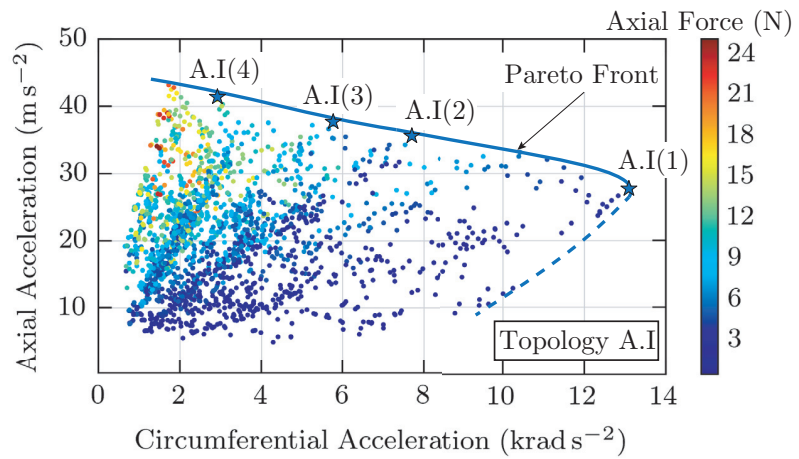

(a)

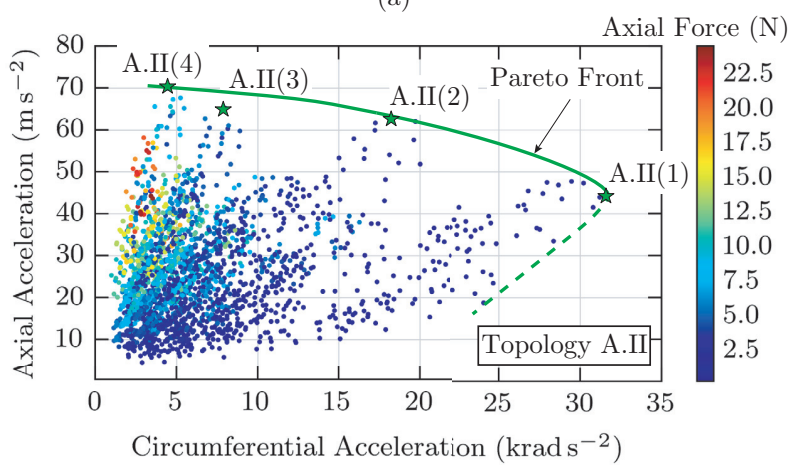

(b)

Fig. 3: Slotless LiRA Topology A performances, where each dot represents one geometry combination. The accomplished axial force $F_{\mathrm{z}}$ is denoted by the color of the dots. (a) Mover with radially magnetized PMs. (b) Mover with Halbach arrangement of PMs.

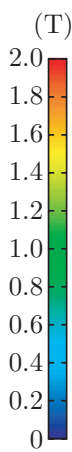

(T)

Fig. 4: Flux density in the mover and stator cores of the Topology A.I(3) candidate design with open slots and semi-closed slots (radial and axial cross sections).

the actuator performance, which is shown in Fig. 9 and will be further discussed in Sec. $\mathbf{V}$ where also a comparison with Topology B candidate designs is conducted. The flux density in the mover and stator cores of the slotted Topology A.I(3) is shown in Fig. 4, where the maximum flux density is below $2 \mathrm{~T}$. 


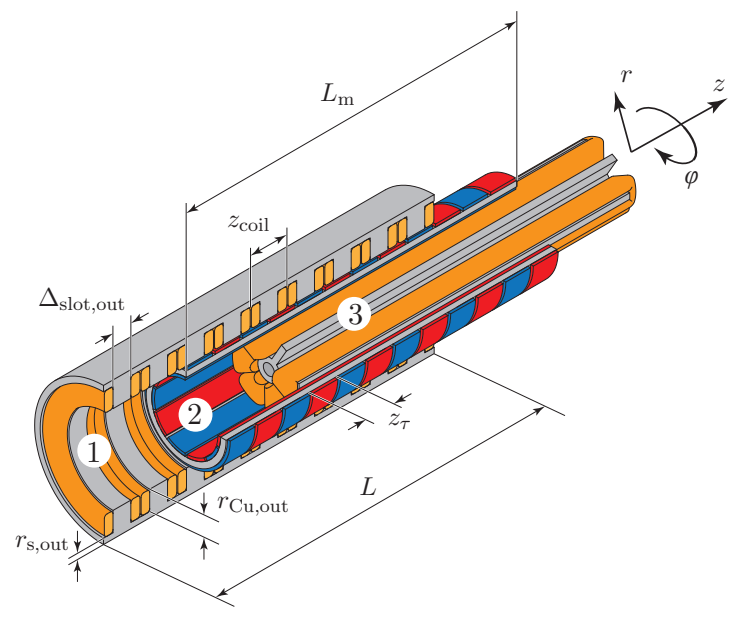

(a)

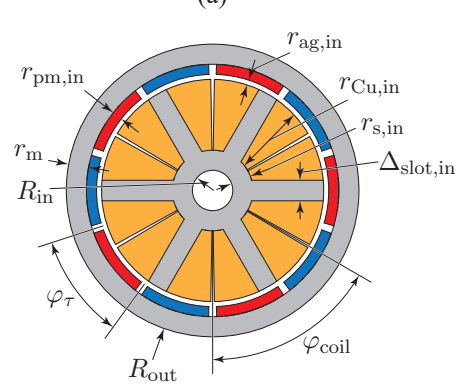

(b)

Fig. 5: LiRA Topology B: (a) 3-D cross-section with geometry parameters in $z$ direction; (1) outer stator, (2) mover, (3) inner stator. (b) Radial cross-section of the inner machine with geometry parameters in $r, \varphi$ directions.

\section{Topology B}

This topology can be best described as the co-axial arrangement of a rotating and a tubular-linear PM machine, and is depicted in Fig. 5(a). For the sake of brevity, only the arrangement with the rotational actuator (RA) being inside and the linear actuator (LA) being outside is considered in this paper, even though the inverse arrangement is also possible. There are two stators, and similarly the mover has two sets of PMs. The back iron in the mover ensures the magnetic decoupling of the inner and outer machine. This means that the machines can be optimized separately, as long as the outer radius of the inner machine and the inner radius of the outer machine are chosen accordingly, their axial lengths are kept the same, and the combined 3-D magnetic flux density is accounted for while dimensioning the back iron of the mover.

Topology A, analyzed in Sec. III, showed a significant improvement of performance when slots were introduced into the slotless stator. Hence, the analysis of Topology B starts with the open slot stator geometry. Geometrical parameters are introduced in Fig. 5 and its discrete design space is defined in Tab. III.

Studying the thermal model of Topology A reveals that the
TABLE III: Topology B discrete geometric parameters set, where: $\varphi_{\tau}=2 \pi / N_{\text {pm,in }}, z_{\tau}=L / N_{\text {pm,out }}, \varphi_{\text {coil }}=2 \pi / N_{\text {coil,in }}, z_{\text {coil }}=$ $L / N_{\text {coil,out }}, K_{\text {slot,in }}=\left(R_{\text {in }}+r_{\text {s,in }}\right) \cdot \varphi_{\text {coil }}, K_{\text {slot,out }}=z_{\text {coil }}$ and $r_{\mathrm{Cu}}=R_{\mathrm{out}}-\left(R_{\mathrm{in}}+r_{\mathrm{s}, \text { in }}+r_{\mathrm{ag}, \text { in }}+r_{\mathrm{pm}, \text { in }}+r_{\mathrm{m}}+r_{\mathrm{pm}, \text { out }}+\right.$ $\left.r_{\text {ag,out }}+r_{\mathrm{s}, \text { out }}\right)$.

\begin{tabular}{|c|c|c|}
\hline Symbol & Parameter name & Values \\
\hline \multicolumn{3}{|c|}{ Swept parameters } \\
\hline$r_{\mathrm{pm}, \mathrm{in}}$ & inner PM thickness & $1,1.5(\mathrm{~mm})$ \\
\hline$r_{\mathrm{pm}, \text { out }}$ & outer PM thickness & $1,1.5(\mathrm{~mm})$ \\
\hline$\varphi_{\mathrm{pm}, \mathrm{in}}$ & inner $\operatorname{PM} \varphi$ size & $0.5,0.75,1 \times \varphi_{\tau}$ \\
\hline$z_{\mathrm{pm}, \mathrm{out}}$ & outer PM $z$ size & $0.5,0.75,1 \times z_{\tau}$ \\
\hline$N_{\mathrm{pm}, \text { in }}$ & pole number, inner & 8,10 \\
\hline$N_{\text {pm,out }}$ & pole number, outer & $8,10,14,16$ \\
\hline$N_{\text {coil,out }}$ & coil number, outer & 12,15 \\
\hline$\Delta_{\text {slot,in }}$ & slot thickness, inner & $0.3,0.5,0.7 \times K_{\text {slot }, \text { in }}$ \\
\hline$\Delta_{\text {slot,out }}$ & slot thickness, outer & $0.4,0.6,0.8 \times K_{\text {slot,out }}$ \\
\hline$r_{\mathrm{Cu}, \text { in }}$ & inner $\mathrm{Cu}$, radial size & $0.4,0.5,0.6 \times r_{\mathrm{Cu}}$ \\
\hline \multicolumn{3}{|c|}{ Fixed parameters } \\
\hline$R_{\text {in }}$ & inner radius & $2 \mathrm{~mm}$ \\
\hline$R_{\text {out }}$ & outer radius & $25 \mathrm{~mm}$ \\
\hline$L$ & actuator length & $120 \mathrm{~mm}$ \\
\hline$r_{\mathrm{s}, \text { in }}$ & inner stator iron thickness & $2 \mathrm{~mm}$ \\
\hline$r_{\mathrm{ag}, \mathrm{in}}$ & inner air gap & $0.5 \mathrm{~mm}$ \\
\hline$r_{\mathrm{m}}$ & mover iron thickness & $2 \mathrm{~mm}$ \\
\hline$r_{\text {ag,out }}$ & outer air gap & $0.5 \mathrm{~mm}$ \\
\hline$r_{\mathrm{s}, \text { out }}$ & outer stator iron thickness & $2 \mathrm{~mm}$ \\
\hline$N_{\text {coil }, \text { in }}$ & coil number, inner & 6 \\
\hline
\end{tabular}

main contributor to the thermal resistance is the stator-to-air thermal resistance $R_{\mathrm{S}-\mathrm{a}}^{\mathrm{T}}$. As the outer dimensions of the LiRA Topologies A and B are the same in this analysis, conclusions regarding the thermal properties of Topology A can be applied also for Topology B. For the outer stator this is obvious. In the case of inner stator, initially the same thermal properties are assumed as for the outer stator. This is justified since the inner and outer stator are assumed to be connected with a mechanical assembly of low thermal resistance.

The inherent magnetic decoupling of this actuator topology allows the use of 2-D FEM models for the analysis. Within the same volume as Topology A, and with the same copper losses, magnetostatic simulations are performed twice for every geometry combination of Topology B where: (1) torque and circumferential acceleration and (2) axial force and acceleration are calculated.

The performances of open slot Topology B, i.e. the achievable circumferential and axial accelerations are shown in Fig. 6. Also here, as with Topology A, acceleration and axial force are in contradiction.

The choice of the optimum geometry depends on the application in which the LiRA is intended to be used, i.e. whether high acceleration or high thrust (axial) force is of primary importance. For this reason, four different Topology B designs are chosen from Fig. 6 (B(1)-B(4)) and discussed in more detail. The geometry combinations that define these designs are given in Tab. IV. In Fig. 7, two designs, one with higher circumferential acceleration $(B(1))$, and one with high axial acceleration (B(4)) are shown. Looking at these two candidate designs, it is possible to see that the PM volume influences the Topology B optimization objectives. In design $\mathrm{B}(4)$, the PM volume is lower in the RA and higher in the LA, which, as a consequence leads to a higher flux linkage in the 


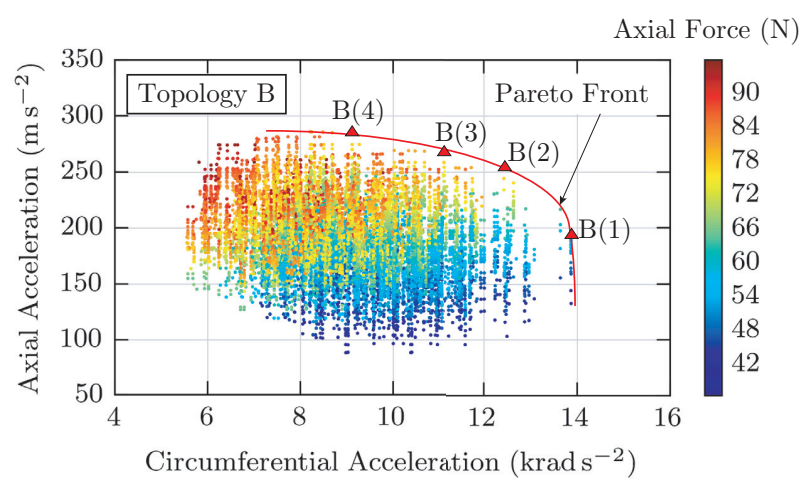

Fig. 6: Performances (circumferential $a_{\varphi}$ and axial $a_{\mathrm{z}}$ accelerations) of actuator Topology B where each dot represents one geometry combination for $25 \mathrm{~W}$ of copper losses. Accomplished axial force $F_{\mathrm{z}}$ is denoted by the color of the dots. The best performances are at the edge of the performance space, bounded by the Pareto Front.

TABLE IV: Parameters of Topology B candidate designs.

\begin{tabular}{ll|ccccl}
\hline \hline & & \multicolumn{7}{|c}{ Top. B Candidate Designs } & \\
Parameter & Unit & $\mathbf{B}(\mathbf{1})$ & $\mathbf{B}(\mathbf{2})$ & $\mathbf{B}(\mathbf{3})$ & $\mathbf{B}(\mathbf{4})$ & \\
\hline$r_{\mathrm{pm}, \text { in }}$ & $(\mathrm{mm})$ & 1 & 1 & 1 & 1 & \\
$r_{\mathrm{pm}, \text { out }}$ & $(\mathrm{mm})$ & 1 & 1 & 1 & 1 & \\
$\varphi_{\mathrm{pm}, \text { in }}$ & - & 1 & 1 & 0.75 & 0.5 & $\times \varphi_{\tau}$ \\
$z_{\mathrm{pm}, \text { out }}$ & - & 0.5 & 0.75 & 0.75 & 0.75 & $\times z_{\tau}$ \\
$N_{\text {pm,in }}$ & - & 8 & 8 & 8 & 8 & \\
$N_{\text {pm,out }}$ & - & 14 & 16 & 16 & 16 & \\
$N_{\text {coil,out }}$ & - & 15 & 12 & 12 & 12 & \\
$\Delta_{\text {slot,in }}$ & - & 0.3 & 0.3 & 0.5 & 0.3 & $\times K_{\text {slot }, \text { in }}$ \\
$\Delta_{\text {slot,out }}$ & - & 0.6 & 0.6 & 0.6 & 0.6 & $\times K_{\text {slot }, \text { out }}$ \\
$r_{\mathrm{Cu}, \text { in }}$ & - & 0.4 & 0.4 & 0.4 & 0.4 & $\times r_{\mathrm{Cu}}$ \\
\hline
\end{tabular}

Topology B Candidate Designs

$(\mathrm{T})$

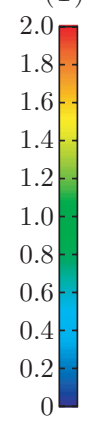

$\begin{array}{r}2.0 \\ 1.8 \\ 1.6 \\ 1.4 \\ 1.2 \\ 1.0 \\ 0.8 \\ 0.6 \\ 0.4 \\ 0.2 \\ 0 \\ 0\end{array}-$.

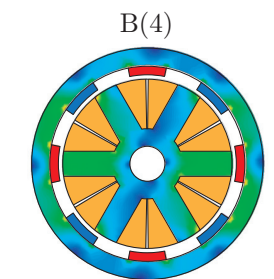

Inner Rotational Actuators

Outer Tubular Linear Actuators
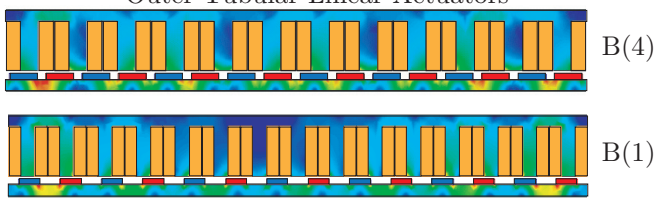

\begin{tabular}{cc|c|c} 
& & $\mathrm{B}(4)$ & $\mathrm{B}(1)$ \\
\cline { 2 - 4 } $\mathrm{PM}$ volume $\left(\mathrm{cm}^{3}\right):$ & Rotational Actuator & 5 & 10 \\
\cline { 2 - 4 } Linear Actuator & 9.6 & 6.4
\end{tabular}

Fig. 7: Topology B candidate designs for high circumferential acceleration applications $\mathrm{B}(1)$ and for high axial force and acceleration applications B(4), chosen from the plot shown in Fig. 6.
Outer Tubular Linear Actuator PM Volume
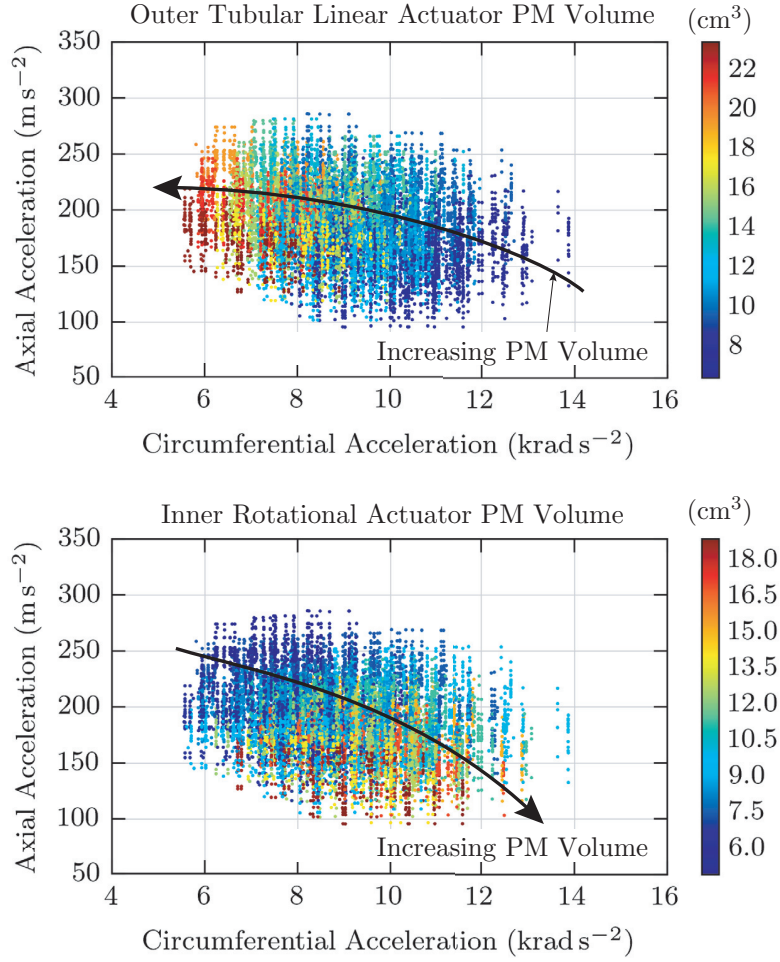

Fig. 8: Performances of actuator Topology B, where the PM volume in the LA (top) and RA (bottom) are denoted with the color of the dots. These plots show the influence of the PM volume on the performance of the actuator.

LA than in the RA. Consequently the LA performs better. In $\mathrm{B}(1)$, contrarily, the RA achieves a better performance. This behavior of Topology B comes from the inherent magnetic decoupling of the inner and outer actuators, where the PMs from one actuator only increase the mass/inertia for the other one, without increasing its force/torque output. Fig. 8 illustrates this property, in which the PM volume of the RA and LA are denoted by the color of the dots.

\section{Comparison of the Topologies A ANd B}

A performance overview of the analyzed machines is given in Fig. 9. The arrows in the plot denote the increase of performance when slots are added into the slotless Topology A designs. In the case of teeth saturation (flux density exceeding $2 \mathrm{~T}$ ), the design is omitted in the plot and denoted with ' $x$ '.

The complete magnetic integration of the linear and rotary actuators in Topology A results in a peculiar property of the windings: coil sides contributing to the torque generation do not contribute to axial force generation, and vice versa. In other words, the active length of the rotational (or linear) actuator is in effect seen as the end windings of the linear (or rotational) actuator. As depicted in Fig. 10, this leads to an unfavorably low ratio of the active length to the end winding length, limiting the torque/force-per-copper-loss rating of this type of actuator. On the other hand, the LA of Topology B features no end windings, while the RA has a higher active 


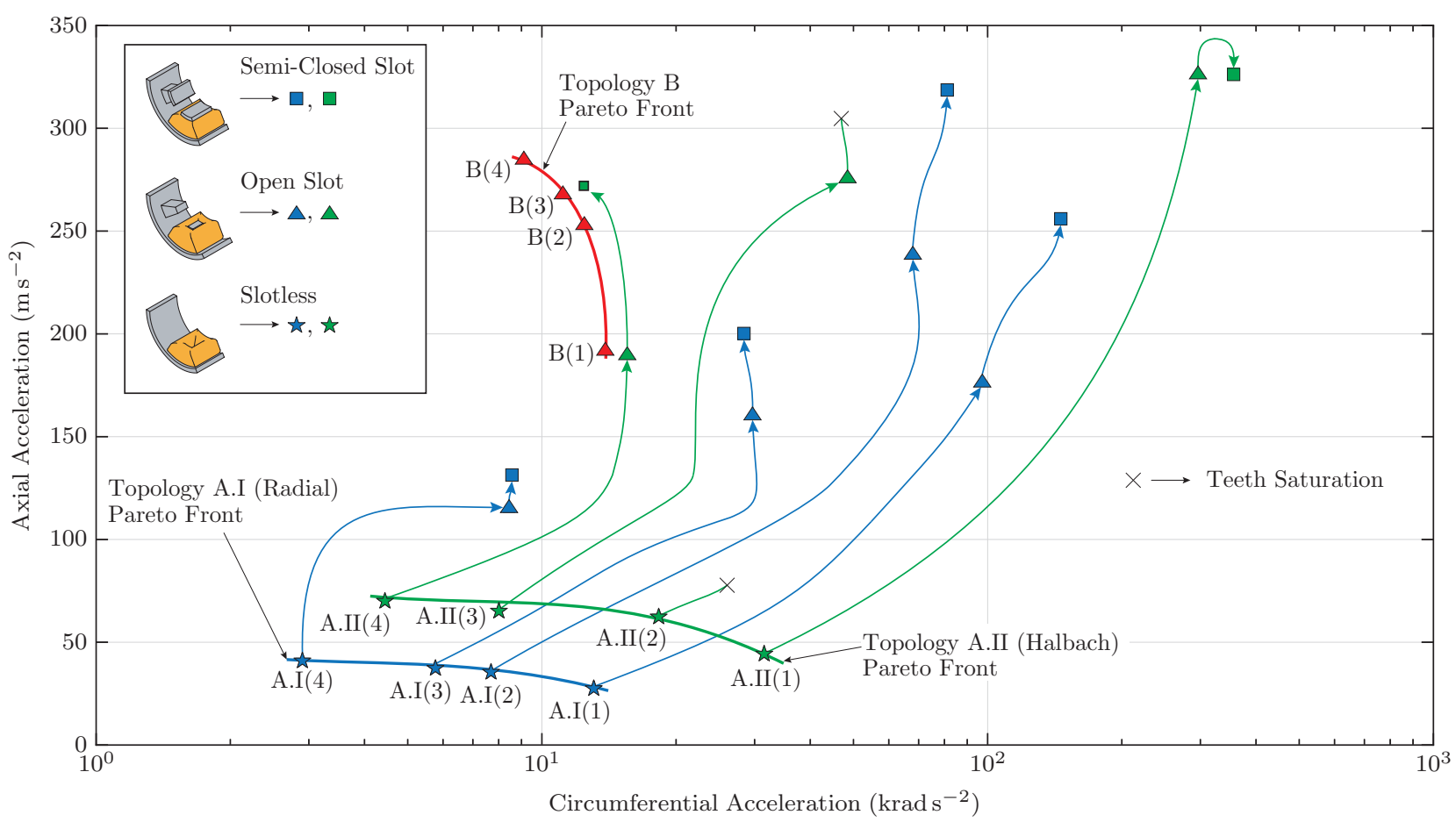

Fig. 9: Comparison of the LiRA Topologies A and B. Changing from the slotless $(\star)$ Topology A to open slots $(\boldsymbol{\Delta})$ and further to semi-closed slots (ם) is denoted with arrows. The performance of the designs with saturated teeth is not shown and/or denoted with ' $\times$ '.

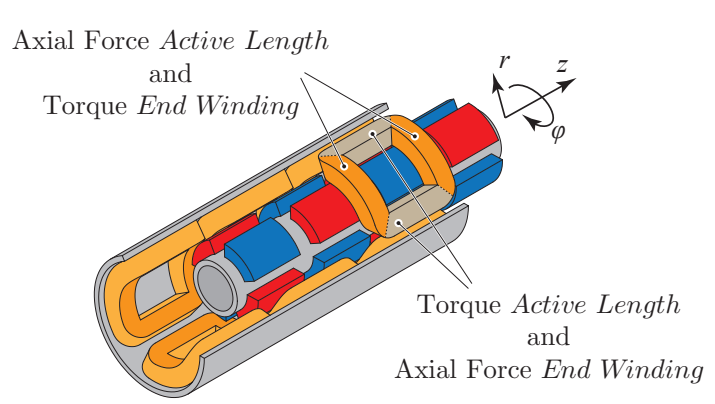

Fig. 10: Topology A active length and end windings.

length to end winding length ratio. As a result, Topology B is capable of generating higher torques and axial forces for the same amount of copper losses. However, the circumferential acceleration capability of Topology $\mathrm{A}$ is higher due to its much lower moment of inertia $J$ of the mover $\left(J \approx R_{\text {mover }}^{4}\right)$ compared to Topology B, where the need for accommodating an inner actuator limits the minimum mover radius. This is shown in Fig. 11, where the torque of Topology B is higher, but the circumferential acceleration is lower compared to Topology A. Similarly, due to the mover's higher mass, Topology B has higher axial force than Topology A, but in terms of axial accelerations they perform alike.

In general, it can be concluded that if the application demands high dynamics with low torque and axial force, Topology A is likely the better choice. On the other hand,
TABLE V: Comparison of the Topologies A and B.

\begin{tabular}{l|cc}
\hline \hline Actuator/Application & Acceleration & Axial Force/Torque \\
\hline Topology A & High & Low \\
Topology B & Low & High \\
\hline
\end{tabular}

if in the application, besides the acceleration, torque and axial force should also be maximized, Topology B is the topology of choice. These conclusions are summarized in Tab. V.

\section{CONCLUSIONS}

Combined rotation and linear movement are desirable in applications such as pick and place machines used for mounting components on PCBs, servo actuation of gearboxes used in automated gear transmissions, and in robotics as arms and end effectors. Linear-Rotary Actuators (LiRA) are used to realize this kind of movement as a direct drive. Their dynamics and compactness highly depend on the chosen actuator topology and design. Therefore, a qualitative evaluation of two LiRA topologies has been carried out in this paper. The aim is to find the LiRA geometry with the highest possible circumferential (rotational) and axial (linear) accelerations. Also, axial force, as well as total actuator mass and volume of the used permanent magnets are analyzed in this evaluation.

A fair comparison of the LiRA topologies is obtained by fixing the outer dimensions (diameter and length) to the same value. The electrical loading is calculated from the established lumped-parameter thermal model. Electromagnetic models of the LiRA topologies are based on the Finite Element 

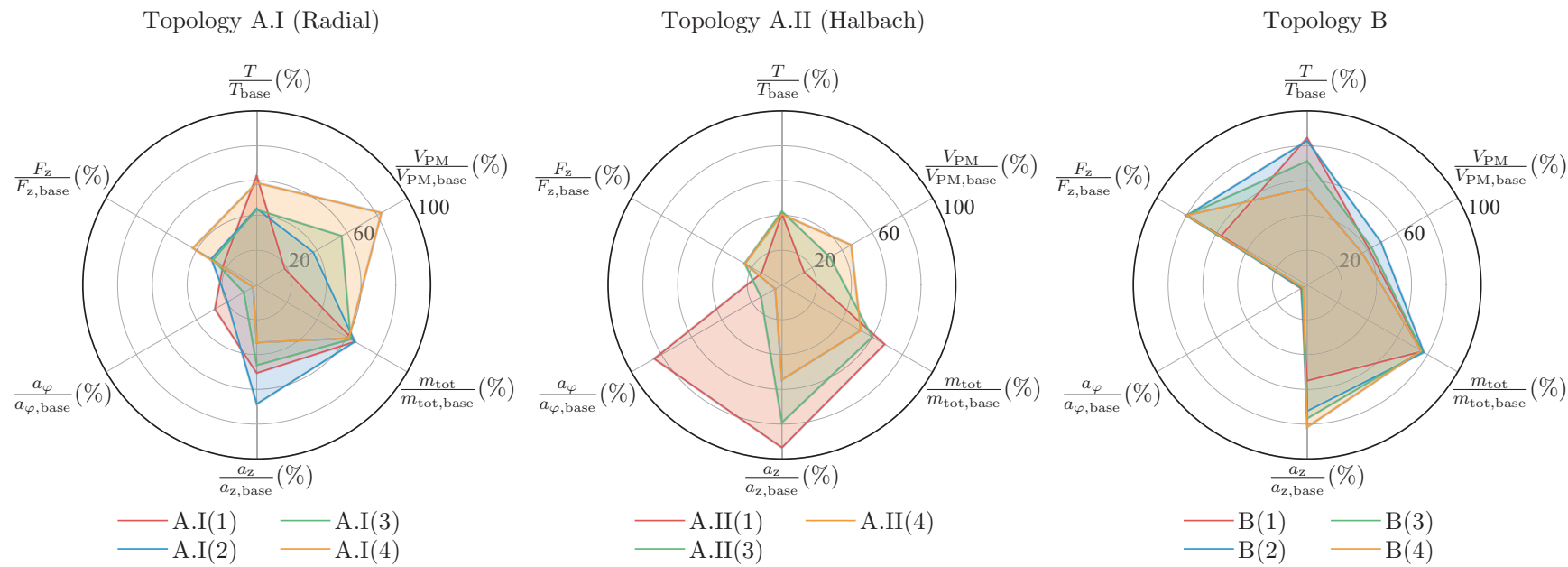

Fig. 11: Comparison of the actuator topologies with open slots: Topology A.I, Topology A.II and Topology B. Base values are equal to: $T_{\text {base }}=700 \mathrm{mN} \mathrm{m}, F_{\mathrm{z}, \text { base }}=100 \mathrm{~N}, a_{\varphi, \text { base }}=350 \mathrm{krad} \mathrm{s}^{-2}, a_{\mathrm{z}, \text { base }}=350 \mathrm{~m} \mathrm{~s}^{-1}, m_{\text {tot, base }}=2 \mathrm{~kg}, V_{\mathrm{PM}, \text { base }}=40 \mathrm{~cm}^{3}$.

Method. In the optimization procedure, for different pole/slot combinations, a discrete set of geometry parameters is defined and then each geometry combination is simulated twice for obtaining torque and axial force, which are then used for calculating circumferential and axial accelerations. After this extensive evaluation, Pareto fronts that enable a qualitative comparison of the two actuator topologies are determined.

One of the LiRA topologies is analyzed with slotless and slotted stators. An improved performance is obtained with the slotted geometry. Finally, it is concluded that the choice of the better LiRA topology depends on the application in which the actuator is used, with one topology offering high torque and axial force, whereas the other one offers higher accelerations, albeit featuring lower torque and axial forces, due to its lower rotor mass and inertia.

\section{ACKNOWLEDGMENT}

The authors would like to acknowledge the support of CADFEM (Suisse) AG concerning the ANSYS software package.

\section{REFERENCES}

[1] K. J. Meessen, J. J. H. Paulides and E. A. Lomonova, "Analysis and Design Considerations of a 2-DoF Rotary-Linear Actuator," in Proc. of IEEE International Electric Machines \& Drives Conference (IEMDC), 2011, pp. 336-341.
[2] A. Turner, K. Ramsay, R. Clark, and D. Howe, "Direct-Drive RotaryLinear Electromechanical Actuation System for Control of Gearshifts in Automated Transmissions," in Proc. of IEEE Vehicle Power and Propulsion Conference, 2007, pp. 267-272.

[3] S. Tanaka, T. Shimono, and Y. Fujimoto, "Optimal Design of Length Factor for Cross-Coupled 2-DoF Motor with Halbach Magnet Array," in Proc. of IEEE International Conference on Mechatronics (ICM), 2015, pp. 529-534.

[4] P. Jin, H. Lin, S. Fang, Y. Yuan, Y. Guo, and Z. Jia, "3-D Analytical Linear Force and Rotary Torque Analysis of Linear and Rotary Permanent Magnet Actuator," IEEE Transactions on Magnetics, vol. 49, no. 7, pp. 3989-3992, 2013

[5] L. Xu, M. Lin, X. Fu, and N. Li, "Analysis of a Double Stator Linear Rotary Permanent Magnet Motor with Orthogonally Arrayed Permanent Magnets," IEEE Transactions on Magnetics, vol. 52, no. 7, 2016.

[6] A. Tüysüz, F. Meyer, M. Steichen, C. Zwyssig, and J. W. Kolar, "Advanced Cooling Methods for High-Speed Electrical Machines," IEEE Transactions on Industry Applications, 2017, to be published.

[7] I. Subotic, C. Gammeter, A. Tüysüz, and J. W. Kolar, "Weight Optimization of an Axial-Flux PM Machine for Airborne Wind Turbines," in Proc. of IEEE International Conference on Power Electronics, Drives \& Energy Systems (PEDES), Trivandrum, India, December 2016.

[8] J. Pyrhönen, T. Jokinen, and V. Hrabovcová, Design of Rotating Electrical Machines. John Wiley \& Sons, 2009, ISBN: 978-1-118-58157-5.

[9] V. T. Morgan, "The Overall Convective Heat Transfer from Smooth Circular Cylinders," Advances in Heat Transfer, vol. 11, pp. 199-264, 1975.

[10] P. Jin, Y. Yuan, G. Jian, H. Lin, S. Fang, and H. Yang, "Static Characteristics of Novel Air-Cored Linear and Rotary Halbach Permanent Magnet Actuator," IEEE Transactions on Magnetics, vol. 50, no. 2, pp. 977-980, 2014. 